\title{
Optical Clearing of Human Skin Using Polyethylene Glycols
}

\author{
Kirill V. Berezin ${ }^{1}$, Konstantin N. Dvoretskiy ${ }^{2 *}$, Vladimir V. Nechaev³, Anatoliy M. Likhter ${ }^{4}$, \\ Ilmira T. Shagautdinova ${ }^{4}$, and Valery V. Tuchin ${ }^{1,5,6}$ \\ ${ }^{1}$ Saratov State University, Saratov 410012, Russia \\ ${ }^{2}$ Saratov State Medical University, Saratov 410012, Russia \\ ${ }^{3}$ Saratov State Technical University, Saratov 410054, Russia \\ ${ }^{4}$ Astrakhan State University, Astrakhan 414056, Russia \\ ${ }^{5}$ Institute of Precision Mechanics and Control, Russian Academy of Sciences, Saratov 410028, Russia \\ ${ }^{6}$ Tomsk State University, Tomsk 634050, Russia \\ *e-mail: dcn@yandex.ru
}

\begin{abstract}
Molecular docking and quantum chemistry (PM6 and DFT/B3LYP) methods have been used to investigate the interaction of a number of biological tissue immersion clearing agents (PEG200, PEG300 and PEG400) with collagen mimetic peptides $(\mathrm{GPH})_{3}$. Correlations between the rate (efficiency) of optical clearing and the energy of complex formation are established. (C) 2020 Journal of Biomedical Photonics \& Engineering.
\end{abstract}

\begin{abstract}
Keywords: molecular dynamics; quantum chemistry; immersion optical clearing.

Paper \#3373 received 23 May 2020; revised manuscript received 11 Jun 2020; accepted for publication 12 Jun 2020; published online 27 Jun 2020. doi: 10.18287/JBPE20.06.020308.
\end{abstract}

\section{Introduction}

The use of modern methods of biomedical optics and photomedicine for the diagnosis and treatment of diseases is experiencing difficulties due to the fact that the skin and many other tissues have strong light scattering in the visible and near-infrared regions. The scattering is caused by the inhomogeneities of the refractive indices at the boundaries of various macromolecular structures, mainly, on collagen fibers, which are mainly responsible for the scattering of light in the skin [1]. These difficulties are overcome by introducing biocompatible molecular agents into the tissue, which contribute, to some extent, to its optical clearing [2-4]. Quite a lot of works was devoted to experimental studies of the optical clearing of various types of tissues in vitro and in vivo [5-11] that indicates the importance of the problem. Mathematical models of light propagation in strongly scattering tissues with absorption are discussed in Ref. [2]. Tuchina et al. [12] considered the effect of model diabetes on the optical clearing of the skin of laboratory mice. Wen et al. [13] explored the mechanism of optical clearing of the skin using glycerol as a clearing agent to improve second harmonic generation (SHG) imaging. However, the mechanisms of optical clearing at the molecular level are still not fully determined, and only a few works [1, 14-17] are devoted to the study of molecular processes responsible for the optical clearing of the skin. Yu et al.
[18] present the results of studies of the dehydrating properties of clearing agents. The authors note that dehydration is only one of the possible mechanisms leading to better optical transparency of tissues. Conducting research in this area opens the way to understanding the essence of optical clearing processes at the molecular level, which, in turn, offers the use of new active clearing agents with desired properties.

In this regard, in order to establish a correlation between the rate (efficiency) of optical clearing of tissue and the parameter of intermolecular interaction (energy of complex formation), the interaction of a number of immersion clearing agents (polyethylene glycols) with collagen mimetic peptides $(\mathrm{GPH})_{3}$ using molecular docking and quantum chemistry methods (PM6 and DFT/B3LYP) is studied.

Due to its effectiveness, availability and biocompatibility, polyethylene glycol (PEG) can be successfully used as an immersion optical clearing agent $[19,20]$. Also, PEG is actively used in medicine and cosmetology as a base for ointments, registered as a food additive E1521, applied as a solvent, extractant, preservative, as well as a strong osmotic.

\section{Molecular Modeling}

We used collagen mimetic peptide $(\mathrm{GPH})_{3}$ [21] that forms the basis of most of the regular domains of human collagen, as the molecular model of collagen. Such 
relatively small synthetic peptides are often used for molecular simulation of collagens. The threedimensional model of the peptide was constructed according to data from the Protein Data Bank (PDB) with the subsequent addition of hydrogen atoms and structure optimization by the molecular dynamics method [22]. We have considered three PEG molecules with a molecular weight of 200,300 and $400 \mathrm{Da}$ as immersion clearing agents (OCAs) for that experimental data on the efficiency of optical clearing (OC) are available in Refs. $[19,20]$. The rate (efficiency) of OC of the skin was estimated in three spectral ranges using the expression

$$
O C_{e f}=\left(\mu_{s 0}-\mu_{s}(t)\right) / \mu_{s 0}
$$

where $\mu_{s 0}$ is the scattering coefficient at the initial time and $\mu_{s}(t)$ is the scattering coefficient value at time $t$ of the optical clearing of the skin. Molecular simulation of the interaction of OCAs with collagen was carried out in two stages.

At the first stage, all the lowest energy conformers of the considered sugars in an isolated state were determined and calculated by the DFT/B3LYP/6$311+\mathrm{G}(\mathrm{d}, \mathrm{p})$ method $[23,24]$ using the Gaussian program [25]. The calculated geometrical parameters were later used in the simulation of these systems within the framework of classical molecular docking. The wavenumbers of vibrational transitions were also calculated, which turned out to be positive that additionally indicates the presence of molecular systems in local minima.

At the second stage, the minimal fragment of the mimetic peptide retaining a regular structure, that is, $\left((\mathrm{GPH})_{3}\right)_{2}$, consisting of 231 atoms, the structure of which was pre-optimized using the semi-empirical method PM6 [26], was used to estimate the intermolecular interaction energy of selected OCAs with collagen. This optimized structure of the collagen model was used for molecular docking with OCAs in the AutoDockVina software [27]. After carrying out molecular docking for each interacting system, the first ten most advantageous configurations were selected, which were further optimized by the semi-empirical PM6 method. Then, the total electron energy of the complexes was calculated by performing a single SCF procedure using the DFT/B3LYP/6-31G(d) method. A similar procedure was used to obtain the total electron energy values of the OCAs and the peptide fragment. The energy of intermolecular interaction was calculated as the difference between the total energies of the complex and the sum of the energies of its individual components. To find out the correlation with the efficiency of OC, we selected the highest values of the intermolecular interaction energies corresponding to the most probable structures of the complexes.

\section{Results and Discussion}

The spatial configurations of the lowest energy isolated conformers of PEGs calculated using the DFT/B3LYP/6 $311+\mathrm{G}(\mathrm{d}, \mathrm{p})$ method are shown in Fig. 1. As can be seen, for all of the compounds presented, the most favorable in the isolated state are conformations in which hydroxyl groups are located as close to each other as possible, which often leads to the formation of intramolecular hydrogen bonds.

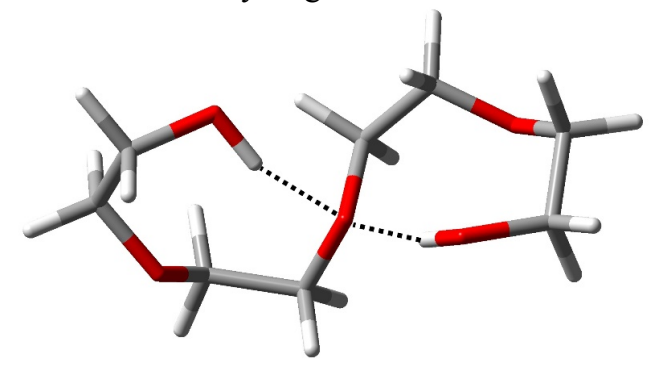

PEG200

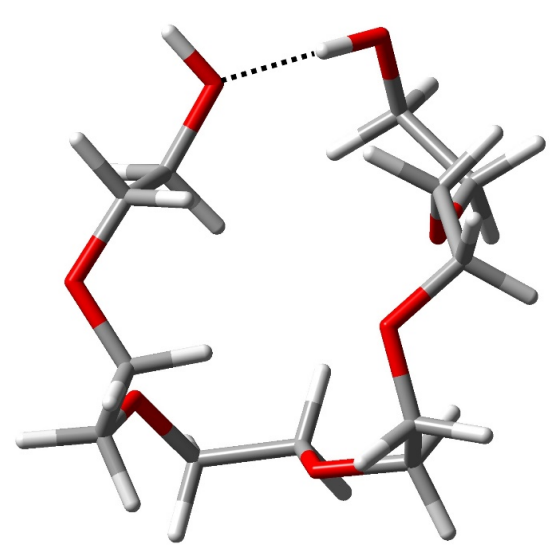

PEG300

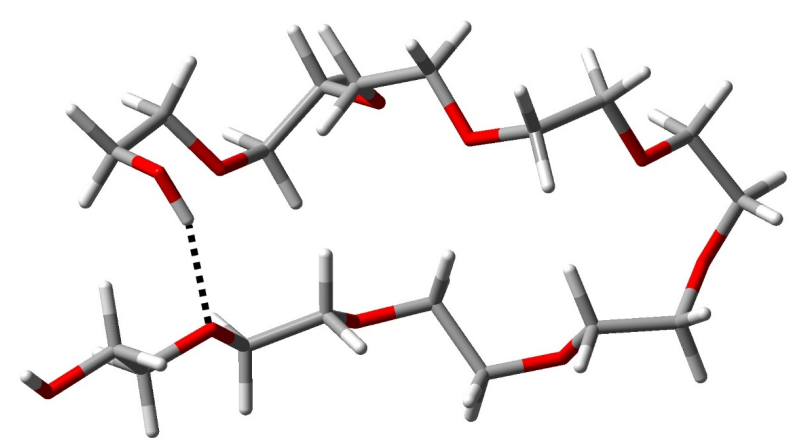

PEG400

Fig. 1 Spatial configurations of the lowest energy isolated conformers of optical clearing agents calculated by DFT/B3LYP/6 311+G(d,p) method. The dotted lines in the figure show intramolecular hydrogen bonds

At the second stage of the simulation, the energy of intermolecular interactions of OCAs with a fragment of the mimetic collagen peptide $-\left((\mathrm{GPH})_{3}\right)_{2}$, the spatial structure of which is shown in Fig. 2, was calculated. 


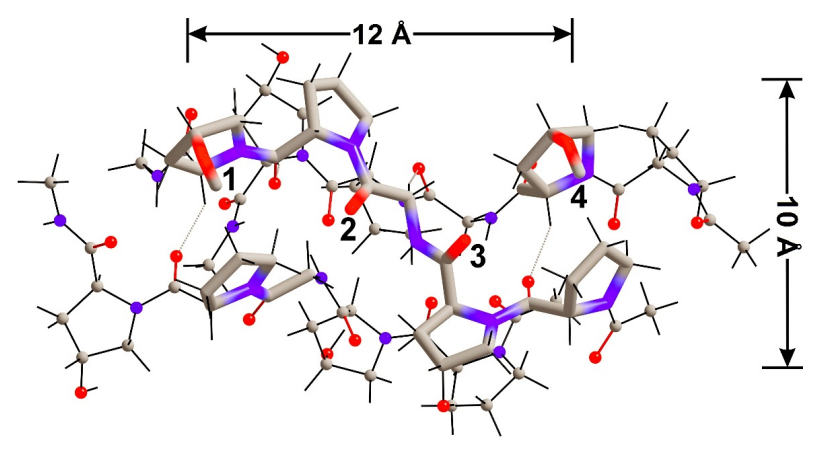

Fig. 2 Spatial structure of the mimetic peptide fragment $-\left((\mathrm{GPH})_{3}\right)_{2}$ optimized in the framework of the semi-empirical PM6 method. Numbers indicate the molecular groups involved in the formation of hydrogen bonds with optical clearing agents. The dotted lines in the Fig. show the hydrogen bonds between different $\alpha$ chains.

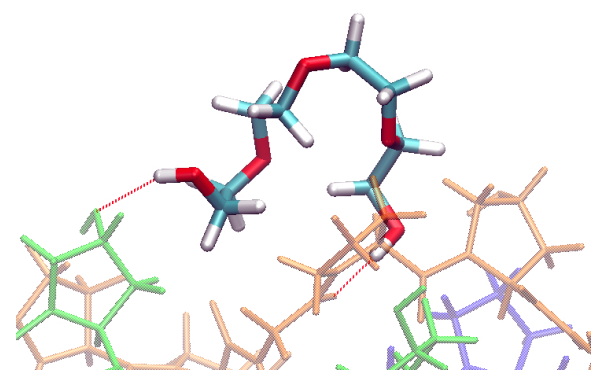

PEG200

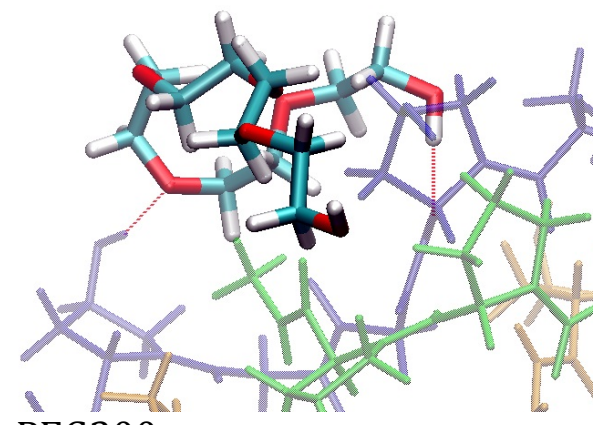

PEG300

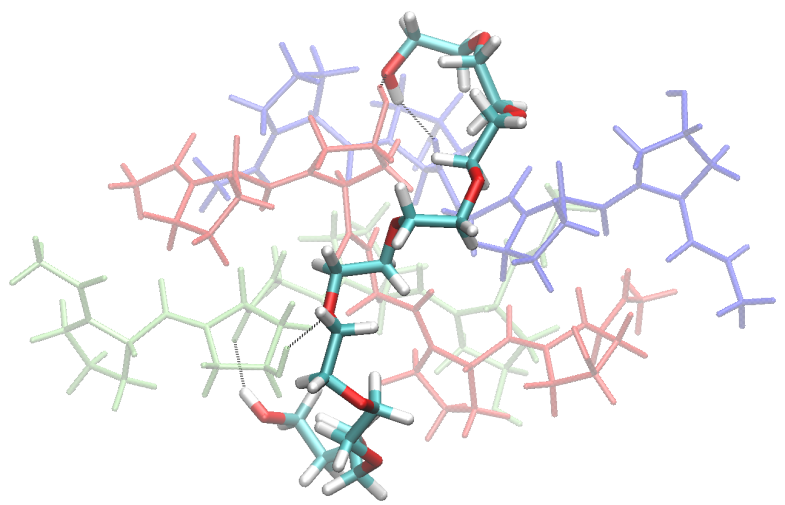

\section{PEG400}

Fig. 3 Structure of the PM6-calculated hydrogen-bound complexes formed by a collagen fragment $\left((\mathrm{GPH})_{3}\right)_{2}$ and immersion optical clearing agents. The dotted line shows classic intermolecular hydrogen bonds.
Table 1 Lengths of hydrogen bonds and energy of intermolecular interactions between a collagen fragment $\left((\mathrm{GPH})_{3}\right)_{2}$ and various clearing agents calculated by the PM6/B3LYP/6-31G(d) method and experimental values of the rate (efficiency) of optical clearing.

\begin{tabular}{|c|c|c|c|}
\hline $\begin{array}{c}\text { Type of } \\
\text { agent }\end{array}$ & $\begin{array}{c}\text { Lengths of } \\
\text { hydrogen } \\
\text { bond, } \AA\end{array}$ & $\Delta \mathrm{E}$ & $\begin{array}{c}\text { Efficiency of } \\
\text { optical clearing }\end{array}$ \\
\hline PEG200 & $2.04 ; 2.06$ & -42.41 & $0.31 \pm 0.10[19]$ \\
\hline PEG300 & $1.83 ; 1.95$ & -48.92 & $0.36 \pm 0.14[20]$ \\
\hline PEG400 & $\begin{array}{c}1.84 ; 1.84 ; \\
1.93 ; 2.09\end{array}$ & -73.91 & $0.42 \pm 0.10[20]$ \\
\hline
\end{tabular}

The molecular capture pocket is a section of a peptide approximately $10 \times 12 \AA$ in size, in which there are four functional groups available for intermolecular binding: two carbonyl groups (one (2) on the glycine residue, the other (3) on the hydroxyproline residue of the same $\alpha$-chain) and two alcohol groups (1 and 4) on the hydroxyproline residues of various $\alpha$-chains (Fig. 2). When collagen interacts with low-molecular agents, a certain spatial adjustment of the molecular pocket occurs to form the largest number of possible hydrogen bonds.

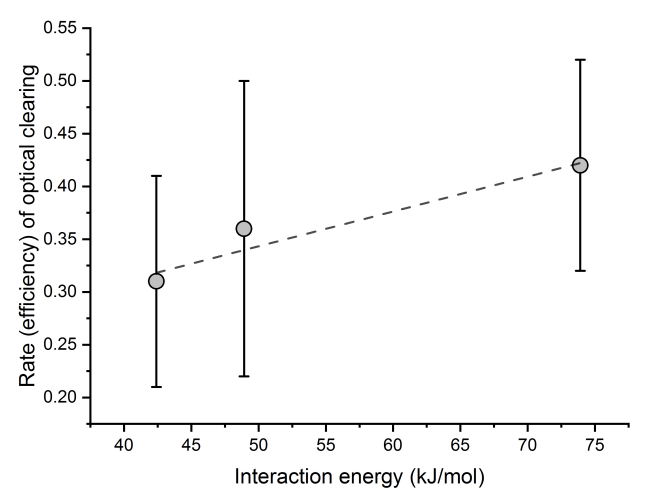

Fig. 4 The dependence of the efficiency of optical clearing of rat skin $[19,20]$ on the energy of interaction of the collagen peptide molecule with the molecules of clearing agents.

Fig. 3 shows the spatial structure of hydrogen-bound complexes formed by collagen fragments $\left((\mathrm{GPH})_{3}\right)_{2}$ and the OCAs. For convenience of discussion of the results obtained, Table 1 summarizes the quantitative parameters of intermolecular interactions (the values of the lengths of classical hydrogen bonds, formed according to the calculation between the active groups of the collagen molecular pocket and the hydroxyl groups of the OCAs, and the calculated energies of the intermolecular interactions). Fig. 3 also shows that the distance between the alcohol groups at the ends of the PEG400 molecule is already sufficient to form hydrogen bonds with different landing molecular pockets on the surface of the molecule. The energy of 
this interaction is much greater than that of the shorter PEG200 and PEG300 molecules, which are able to form hydrogen bonds only within one molecular capture pocket. From the data it follows that the dependence of the efficiency of $\mathrm{OC}$ of the skin of ex vivo white laboratory rats $[19,20]$ correlates well with the interaction energy calculated by the PM6/B3LYP/6-31G method for the selected OCAs (Fig. 4). The linear correlation coefficient is 0.94 . This suggests the fundamental importance of the post-diffusion stage, in which the interaction of collagen with OCAs occurs, and its effect on the $\mathrm{OC}$ of tissues takes place. The results of the study suggest that in the process of such an interaction, a partial replacement of water associated with collagen occurs. This leads to the destruction of the network of hydrogen bonds and, as a result, to the reversible process of dissolution of collagen fibrils, which in turn decreases their refractive index and matches it with the intercollagen medium. The higher the affinity of the OCA to collagen, the more effective is this process.

The next fundamental step to increase the efficiency of interaction may be the selection of a molecular agent with such structural characteristics that would allow it to interact with two or more molecular pockets of collagen at once. Such an active OCA can serve as a polymertype molecular system consisting, for example, of sixmembered monosaccharides connected by a mobile carbon-oxygen chain of such a length that saturated rings of sugar fall into the regions of the molecular pockets of collagen and interact with them through their hydroxyl groups. A significant increase in the size of molecules used as OCAs leads to an increase in the viscosity of a substance and, as a consequence, to a decrease in its diffusion coefficient in tissue and an increase in the time of its washing out from tissues. Therefore, the choice of the optimal OCA is a trade-off between the effectiveness of the OC and OCA diffusion time.

Thus, the construction of an adequate molecular model and finding the correlation described above for various optical clearing agents $[7,17,28]$ allows one to predict the optical clearing potential of various molecular systems and select the most effective ones before expensive ex vivo and in vivo animal studies and further for practical use in medicine.

\section{Conclusions}

As a result of complex molecular modeling of the interaction of a number of immersion optical clearing agents with a mimetic collagen peptide $\left((\mathrm{GPH})_{3}\right)_{2}$ correlations between the rate (efficiency) of optical clearing and such a characteristic as the energy of intermolecular interaction of clearing agents with a fragment of a collagen peptide are established.

\section{Disclosures}

All authors declare that there is no conflict of interests in this paper.

\section{Acknowledgments}

V. V. Tuchin was supported by RFBR grant no 18-52-16025.

\section{References}

1. J. M. Hirshburg, Chemical agent induced reduction of skin light scattering: doctoral dissertation, Texas A\&M University (2009).

2. V. V. Tuchin, Tissue Optics: Light Scattering Methods and Instruments for Medical Diagnostics, 3rd ed., PM254, SPIE Press, Bellingham, WA (2015).

3. D. Zhu, K. V. Larin, Q. Luo, and V. V. Tuchin, "Recent progress in tissue optical clearing," Laser \& Photonics Reviews 7(5), 732-757 (2013).

4. E. A. Genina, A. N. Bashkatov, Y. P. Sinichkin, I. Yu. Yanina, and V. V. Tuchin, "Optical clearing of biological tissues: prospects of application in medical diagnostics and phototherapy [Review]," Journal of Biomedical Photonics \& Engineering 1(1), 22-58 (2015).

5. K. V. Larin, M. G. Ghosn, A. N. Bashkatov, E. A. Genina, N. A. Trunina, and V. V. Tuchin, "Optical clearing for OCT image enhancement and in-depth monitoring of molecular diffusion," IEEE Journal of Selected Topics in Quantum Electronics 18(3), 1244-1258 (2012).

6. V. V. Tuchin, "Polarized light interaction with tissues," Journal of Biomedical Optics 21(7), 071114 (2016).

7. A. N. Bashkatov, K. V. Berezin, K. N. Dvoretskiy, M. L. Chernavina, E. A. Genina, V. D. Genin, and V. I. Kochubey, "Measurement of tissue optical properties in the context of tissue optical clearing," Journal of Biomedical Optics 23(9), 091416 (2018).

8. E. N. Lazareva, P. Dyachenko (Timoshina), A. Bucharskaya, N. Navolokin, and V. Tuchin, "Estimation of dehydration of skin by refractometric method using optical clearing agents," Journal of Biomedical Photonics \& Engineering 5(2), 020305 (2019).

9. A. Yu. Sdobnov, M. E. Darvin, E. A. Genina, A. N. Bashkatov, J. Lademann, and V. V. Tuchin, "Recent progress in tissue optical clearing for spectroscopic application," Spectrochimica Acta Part A: Molecular and Biomolecular Spectroscopy 197, 216-229 (2018). 
10. S. M. Zaytsev, Y. I. Svenskaya, E. V. Lengert, G. S. Terentyuk, A. N. Bashkatov, V. V. Tuchin, and E. A. Genina, "Optimized skin optical clearing for optical coherence tomography monitoring of encapsulated drug delivery through the hair follicles," Journal of Biophotonics 13(4), e201960020 (2020).

11. N. Sudheendran, M. Mohamed, M. G. Ghosn, V. V. Tuchin, and K. V. Larin, "Assessment of tissue optical clearing as a function of glucose concentration using optical coherence tomography," Journal of Innovative Optical Health Sciences 3(3), 169-176 (2010).

12. D. K. Tuchina, R. Shi, A. N. Bashkatov, E. A. Genina, D. Zhu, Q. Luo, and V. V. Tuchin, "Ex vivo optical measurements of glucose diffusion kinetics in native and diabetic mouse skin," Journal of Biophotonics 8(4), 332$346(2015)$.

13. X. Wen, Z. Mao, Z. Han, V. V. Tuchin, and D. Zhu, "In vivo skin optical clearing by glycerol solutions: mechanism," Journal of Biophotonics 3(1-2), 44-52 (2010).

14. J. M. Hirshburg, K. M. Ravikumar, W. Hwang, and A. T. Yeh, "Molecular basis for optical clearing of collagenous tissues," Journal of Biomedical Optics 15(5), 055002 (2010).

15. W. Feng, R. Shi, N. Ma, D. K. Tuchina, V. V. Tuchin, and D. Zhu, "Skin optical clearing potential of disaccharides," Journal of Biomedical Optics 21(8), 081207 (2016).

16. K. N. Dvoretsky, K. V. Berezin, M. L. Chernavina, A. M. Likhter, I. T. Shagautdinova, E. M. Antonova, O. N. Grechukhina, and V. V. Tuchin, "Molecular Modeling of the Post-Diffusion Stage of Surface Bio-Tissue Layers Immersion Optical Clearing," Journal of Surface Investigation: X-ray, Synchrotron and Neutron Techniques 12 (5), 961-967 (2018).

17. K. V. Berezin, K. N. Dvoretski, M. L. Chernavina, A. M. Likhter, V. V. Smirnov, I. T. Shagautdinova, E. M. Antonova, E. Yu. Stepanovich, E. A. Dzhalmuhambetova, and V. V. Tuchin, "Molecular modeling of immersion optical clearing of biological tissues," Journal of Molecular Modeling 24(2), 45 (2018).

18. T. Yu, X. Wen, V. V. Tuchin, Q. Luo, and D. Zhu, "Quantitative analysis of dehydration in porcine skin for assessing mechanism of optical clearing," Journal of Biomedical Optics 16(9), 095002 (2011).

19. V. D. Genin, P. Rakotomanga, S. M. Zaytsev, E. A. Genina, E. N. Lazareva, G. Khairallah, M. Amouroux, C. Soussen, H. Chen, W. Feng, D. Zhu, A. N. Bashkatov, W. Blondel, and V. V. Tuchin, "Research and development of effective optical technologies for diagnostics in dermatology," Proceedings of SPIE 11065, 1106505 (2019).

20. D. K. Tuchina, V. D. Genin, A. N. Bashkatov, E. A. Genina, and V. V. Tuchin, "Optical clearing of skin tissue ex vivo with polyethylene glycol," Optics and Spectroscopy 120(1), 36-45 (2016).

21. K. Okuyama, K. Miyama, K. Mizuno, and H. P. Bächinger, "Crystal structure of (Gly-Pro-Hyp)9: implications for the collagen molecular model," Biopolymers 97(8), 607-616 (2012).

22. W. D. Cornell, P. Cieplak, C. I. Bayly, I. R. Gould, K. M. Merz, D. M. Ferguson, D. C. Spellmeyer, T. Fox, J. W. Caldwell, and P. A. Kollman, "A second generation force field for the simulation of proteins, nucleic acids, and organic molecules," Journal of the American Chemical Society 117(19), 5179-5197 (1995).

23. A. D. Becke, "Density - functional thermochemistry. III. The role of exact exchange," The Journal of Chemical Physics 98(7), 5648-5652 (1993).

24. C. Lee, W. Yang, and R. G. Parr, "Development of the Colle-Salvetti correlation-energy formula into a functional of the electron density," Physical Review B 37(2), 785-789 (1988).

25. M. J. Frisch, G. Trucks, H. B. Schlegel, G. E. Scuseria, M. A. Robb, J. R. Cheeseman, G. Scalmani, V. Barone, B. Mennucci,G. A. Petersson, H. Nakatsuji, X. Li, M. Caricato, X. Li, J. Bloino, H. P. Hratchian, A. F. Izmaylov, J. L. Sonnenberg, G. Zheng, M. Hada, and D. J. Fox, Gaussian09, Revision A.02, Gaussian Inc., Wallingford CT (2009).

26. J. J. P. Stewart, "Optimization of parameters for semiempirical methods V: Modification of NDDO approximations and application to 70 elements," Journal of Molecular Modeling 13(12), 1173-1213 (2007).

27. O. Trott, A. J. Olson, “AutoDock Vina: Improving the speed and accuracy of docking with a new scoring function, efficient optimization, and multithreading," Journal of Computational Chemistry 31(2), 455-461 (2010).

28. K. V. Berezin, K. N. Dvoretskii, M. L. Chernavina, V. V. Nechaev, A. M. Likhter, I. T. Shagautdinova, E. M. Antonova, and V. V. Tuchin, "Optical clearing of human skin using some monosaccharides in vivo," Optics and Spectroscopy 127(2), 352-358 (2019). 